Article

\title{
Analysis of Hepatic Fibrosis Characteristics in Cirrhotic Patients with and without Hepatocellular Carcinoma by FTIR Spectral Imaging
}

\author{
Johanna Moreau ${ }^{1,2,+}$, Pascaline Bouzy ${ }^{1,+}{ }^{\dagger}$, Julien Guillard ${ }^{1}{ }^{\circledR}$, Valérie Untereiner ${ }^{3}{ }^{\circledR}$, \\ Roselyne Garnotel ${ }^{1,4}$, Aude Marchal ${ }^{1,5}$, Cyril Gobinet ${ }^{1}$, Christine Terryn ${ }^{3} \mathbb{D}$, \\ Ganesh D. Sockalingum ${ }^{1, \ddagger}$ and Gérard Thiéfin ${ }^{1,2, *, \ddagger(\mathbb{D})}$ \\ 1 Université de Reims Champagne-Ardenne, BioSpecT-EA7506, UFR de Pharmacie, 51097 Reims, France; \\ jhoannam@hotmail.fr (J.M.); pascaline.bouzy@gmail.com (P.B.); julien.guillard@univ-reims.fr (J.G.); \\ roselyne.garnotel@univ-reims.fr (R.G.); amarchal@chu-reims.fr (A.M.); cyril.gobinet@univ-reims.fr (C.G.); \\ ganesh.sockalingum@univ-reims.fr (G.D.S.) \\ 2 Service d'Hépato-Gastroentérologie et de Cancérologie Digestive, Centre Hospitalier Universitaire de Reims, \\ 51092 Reims, France \\ 3 Université de Reims Champagne-Ardenne, Plateforme en Imagerie Cellulaire et Tissulaire (PICT), \\ 51097 Reims Cedex, France; valerie.untereiner@univ-reims.fr (V.U.); christine.terryn@univ-reims.fr (C.T.) \\ 4 Laboratoire de Biochimie-Pharmacologie-Toxicologie, Centre Hospitalier Universitaire de Reims, \\ 51092 Reims, France \\ 5 Service d'Anatomie et Cytologie Pathologique, Centre Hospitalier Universitaire de Reims, \\ 51100 Reims, France \\ * Correspondence: gthiefin@chu-reims.fr; Tel.: +33-6-87517-344; Fax: +33-3-26788-836 \\ + These authors contributed equally to this work. \\ $\ddagger \quad$ These authors contributed equally to study design, conception and supervision.
}

Academic Editors: Kamilla Malek and Derek J. McPhee

Received: 20 July 2020; Accepted: 4 September 2020; Published: 7 September 2020

\begin{abstract}
The evolution of cirrhosis is marked by quantitative and qualitative modifications of the fibrosis tissue and an increasing risk of complications such as hepatocellular carcinoma (HCC). Our purpose was to identify by FTIR imaging the spectral characteristics of hepatic fibrosis in cirrhotic patients with and without HCC. FTIR images were collected at projected pixel sizes of 25 and $2.7 \mu \mathrm{m}$ from paraffinized hepatic tissues of five patients with uncomplicated cirrhosis and five cirrhotic patients with HCC and analyzed by k-means clustering. When compared to the adjacent histological section, the spectral clusters corresponding to hepatic fibrosis and regeneration nodules were easily identified. The fibrosis area estimated by FTIR imaging was correlated to that evaluated by digital image analysis of histological sections and was higher in patients with HCC compared to those without complications. Qualitative differences were also observed when fibrosis areas were specifically targeted at higher resolution. The partition in two clusters of the fibrosis tissue highlighted subtle differences in the spectral characteristics of the two groups of patients. These data show that the quantitative and qualitative changes of fibrosis tissue occurring during the course of cirrhosis are detectable by FTIR imaging, suggesting the possibility of subclassifying cirrhosis into different steps of severity.
\end{abstract}

Keywords: FTIR imaging; k-means; fibrosis; collagen; cirrhosis; hepatocellular carcinoma

\section{Introduction}

Cirrhosis is defined as an advanced stage of diffuse liver fibrosis in response to a chronic injury causing lobular disorganization with formation of fibrous septa and nodules of morphologically and 
functionally abnormal hepatocytes [1]. Classical histological scoring systems such as the Metavir [2] or Ishak systems [3] define cirrhosis as the ultimate stage of hepatic fibrosis but do not differentiate cirrhosis at different stages of severity. A subclassification of cirrhosis would be of clinical interest since cirrhosis at an early stage is potentially reversible when the causal factor is removed, whereas advanced cirrhosis may become irreversible [4] and progress to complications related to portal hypertension and hepatocellular insufficiency. There is also a risk of transformation into hepatocellular carcinoma (HCC). The identification of markers of advanced cirrhosis could allow a subclassification of cirrhotic patients, making it possible to select cirrhotic patients at higher risk and justifying a closer surveillance program.

A histological scoring system, the Laennec system [5,6], has been proposed to subclassify cirrhosis into three categories of severity according to the width of fibrous septa and size of nodules. Using this system, it has been shown that increasing severity of cirrhosis was tightly correlated with both the clinical stage of cirrhosis and the grade of portal hypertension [7]. In addition, an increasing incidence of liver related events has been reported according to the severity of cirrhosis as assessed by this scoring system [8,9]. In particular, the incidence of hepatic decompensation and HCC significantly correlated with the histological subclassification of cirrhosis [9]. However, this scoring system is semi-quantitative and not routinely used.

The molecular and structural characteristics of the extracellular matrix (ECM) vary during the dynamic process of hepatic fibrogenesis $[4,10]$. The production and the relative amounts of different types of collagen change during fibrosis development [4,11]. In parallel, the expression of tissue inhibitors of metalloproteinases (TIMPs) is upregulated and the expression of metalloproteinases (MMPs) decreased, thus favoring an imbalance between MMPs and TIMPs and facilitating the development of hepatic fibrosis [4,12]. Other ECM components may play a role in the progression of liver fibrosis. It has been shown that elastin monomers accumulate in liver tissue during fibrogenesis as a result of overproduction by myofibroblasts and a failure of its degradation by MMP12 [4]. In addition, post-translational modifications of collagen result in the formation of covalent intermolecular cross-links such as pyridinoline, which is involved in the stabilization of collagen at a late stage in the fibrotic process $[13,14]$.

Spectral histopathology, using Fourier transform infrared (FTIR) imaging, provides a "vibrational fingerprint" of the tissue that reflects its molecular and structural composition [15]. Since the ECM molecular profile varies during hepatic fibrogenesis, we hypothesized that spectral analysis of fibrous septa in cirrhotic patients could allow a subclassification of cirrhosis, differentiating uncomplicated and complicated stages of the disease. It is well established that the different types of collagen have a specific spectral signature [16-18]. In addition, FTIR spectroscopic studies of collagen characteristics in rat tail tendons have revealed molecular changes related to the formation of collagen crosslinks during the aging of these tendons $[19,20]$. Previous studies have reported that FTIR imaging was able to differentiate regeneration nodules and fibrotic septa in human cirrhotic samples [21-23] and detect chemical modifications due to diabetes [24]. Thus, analysis of the FTIR spectral characteristics of hepatic fibrosis may have the potential to distinguish patients at different stages of cirrhosis.

The objective of this study was to analyze the spectral characteristics of hepatic fibrosis by FTIR imaging in cirrhotic patients, enabling the differentiation of patients with cirrhosis complicated by HCC and cirrhotic patients without complications.

\section{Results and Discussion}

\subsection{Analysis of Spectral Images of Whole Hepatic Samples Acquired with a Projected Pixel Size of $25 \mu \mathrm{m}$}

Histological and spectral images of two hepatic samples from patients with uncomplicated cirrhosis and cirrhosis complicated by HCC are presented as examples in Figures 1 and 2.

Spectral images were partitioned into 2, 3, 4 and 5 clusters using the k-means method (Figures $1 \mathrm{C}-\mathrm{F}$ and $2 \mathrm{C}-\mathrm{F}$ ). 
A

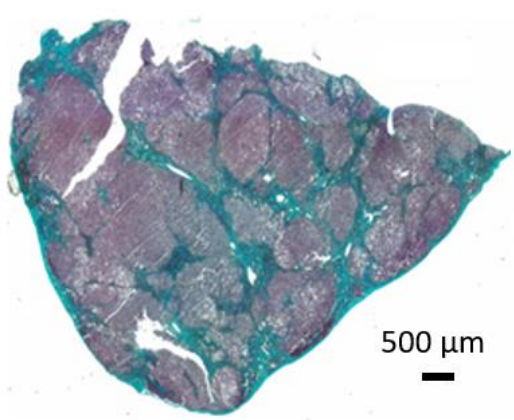

C

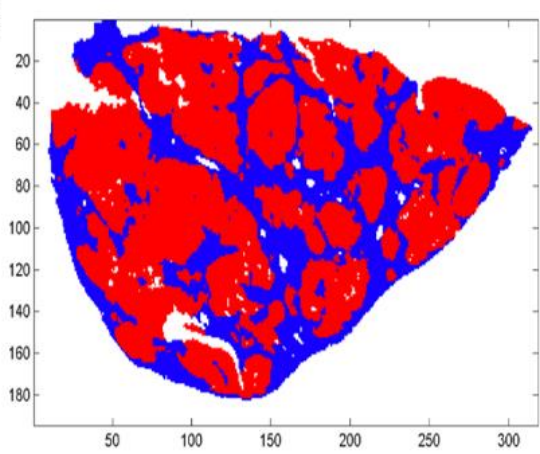

E

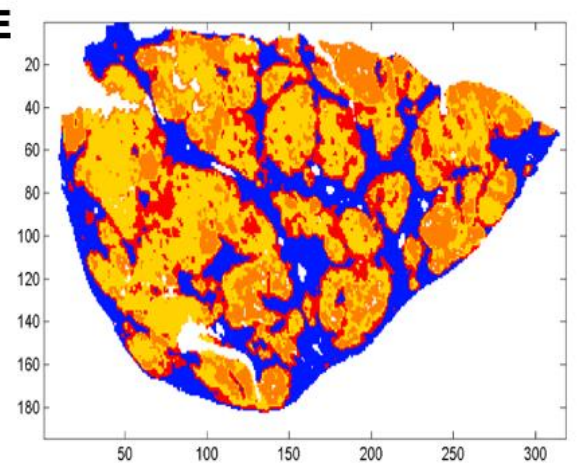

B

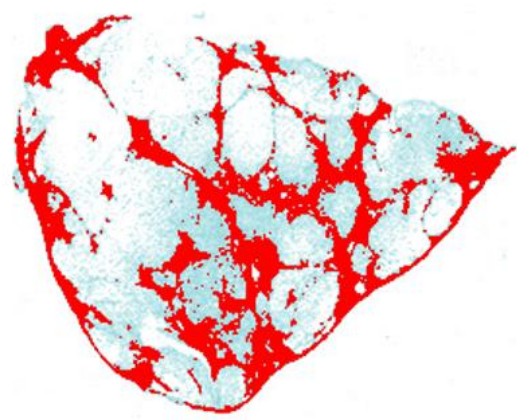

D

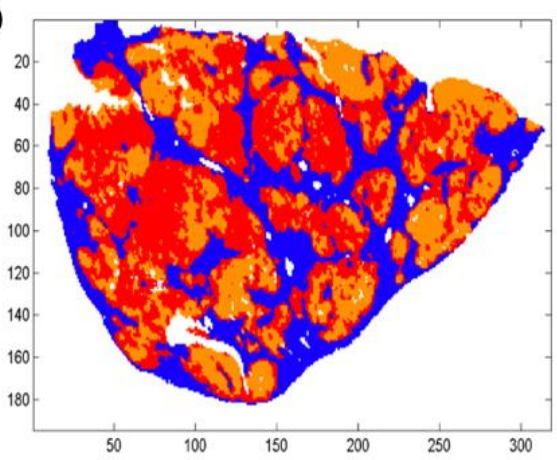

$\mathbf{F}$

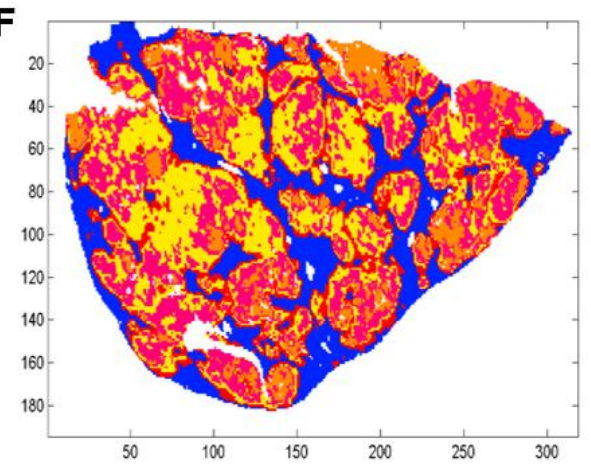

Figure 1. Example of histological and FTIR spectral images of an uncomplicated cirrhosis biopsy. (A) Histological section stained with Masson's trichrome stain showing fibrosis in green. (B) Digital image analysis with fibrosis area in red. (C-F) K-means clustering of FTIR spectral images of an adjacent section in 2, 3, 4 and 5 clusters respectively from a $900-1800 \mathrm{~cm}^{-1}$ infrared absorbance dataset acquired with a projected pixel size of $25 \mu \mathrm{m}$. Clusters are displayed using random pseudo-colors. Fibrosis is represented by the dark blue cluster. Other colors correspond to regeneration nodules.

When compared to the adjacent histological section, the spectral classes corresponding to hepatic fibrosis and regeneration nodules were easily identified. Fibrosis tissue segregated out of nodules in the two-class partition and further partition delineated additional spectral subgroups, mainly in regeneration nodules. In contrast, fibrosis tissue remained homogeneous or mildly heterogeneous in up to two spectral classes compared to nodules as shown in Figures 1C-F and 2C-F. The correlation analysis between fibrosis area percentages estimated by FTIR image clustering and by digital image analysis (DIA) was conducted after exclusion of an outlier (the point corresponding to a fibrosis area of $60-70 \%$ ) in order to avoid a biased statistical analysis. The percentages of fibrosis area as estimated by FTIR image clustering with 3, 4 and 5 classes were significantly correlated to those evaluated by DIA of the corresponding histological sections stained with Masson's trichrome as shown in Figures 1B and 2B. The correlation coefficient $r$ varied between 0.83 and 0.89 ( $p$-value between 0.0012 and 0.0054 ) as shown in Figure 3. A similar tendency was observed when fibrosis areas were estimated by FTIR 
image clustering with two classes but the correlation did not reach the level of significance $(r=0.64$, $p=0.06$ ). For comparison, the scatter plots obtained using the whole dataset including the outlier are shown in the Supplementary Figure S1. The bias due to the outlier point explains the higher level of correlation. Altogether, our data suggest that FTIR image clustering with at least three classes may correctly differentiate between fibrosis tissue and regenerative nodules. However, this study was not designed to develop a predictive model. It is a proof-of-concept study and further work on a larger set of hepatic samples is necessary to build a classification model that would allow an automatic evaluation of fibrosis area without the need for stained adjacent histological sections.

A

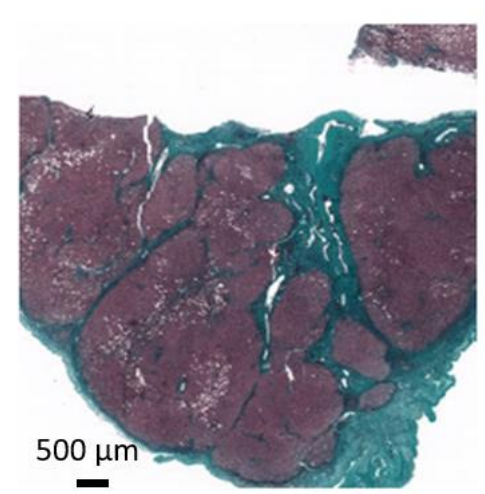

C

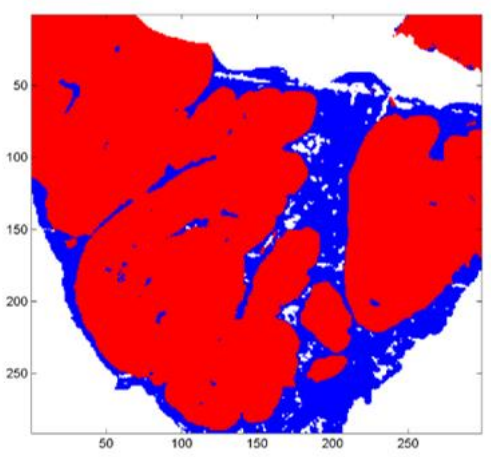

E

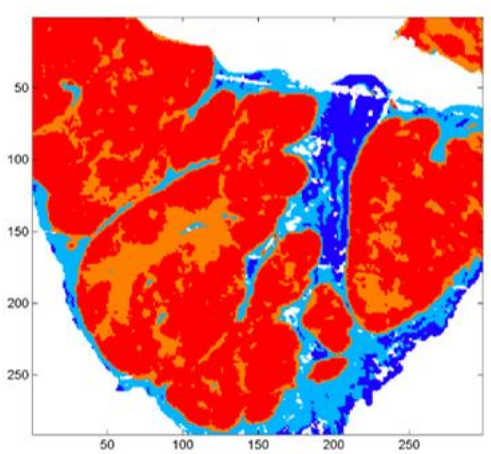

B

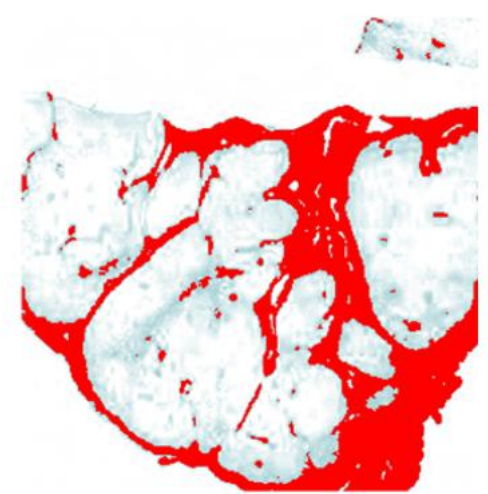

D

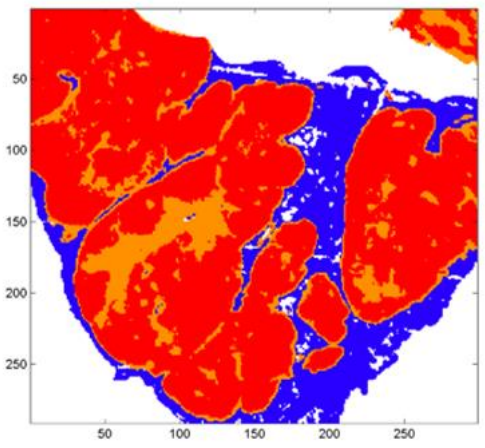

F

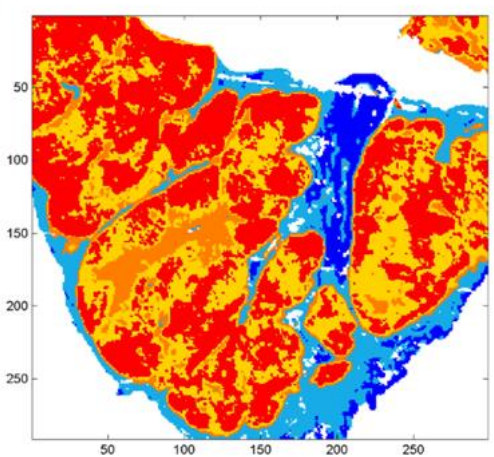

Figure 2. Example of histological and FTIR spectral images of a cirrhosis biopsy from a patient with hepatocellular carcinoma. (A) Histological section stained with Masson's trichrome stain showing fibrosis in green. (B) Digital image analysis with fibrosis area in red. (C-F) K-means clustering of FTIR spectral images of an adjacent section in 2, 3, 4 and 5 clusters respectively from a $900-1800 \mathrm{~cm}^{-1}$ infrared absorbance dataset acquired with a projected pixel size of $25 \mu \mathrm{m}$. Clusters are displayed using random pseudo-colors. Fibrosis is represented by the dark blue cluster in C, D and in dark blue and light blue in $\mathrm{E}$ and $\mathrm{F}$. Other colors correspond to regeneration nodules. 
A

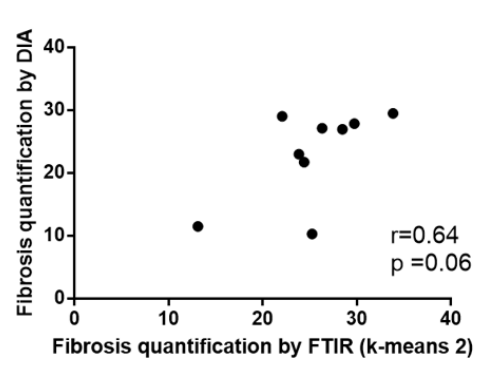

C

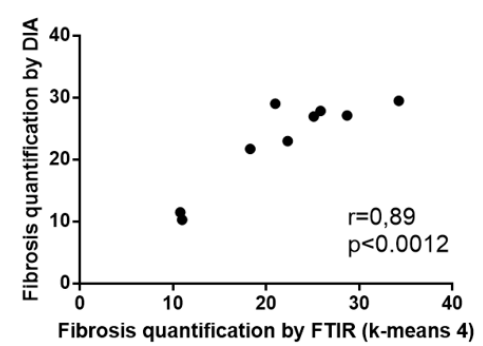

B

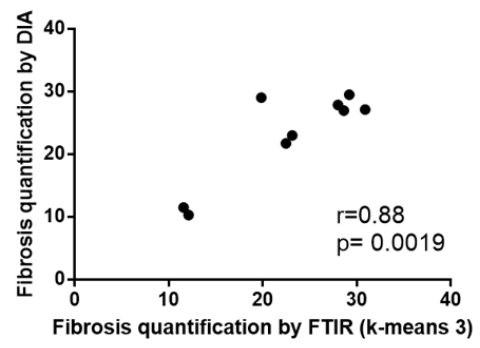

D

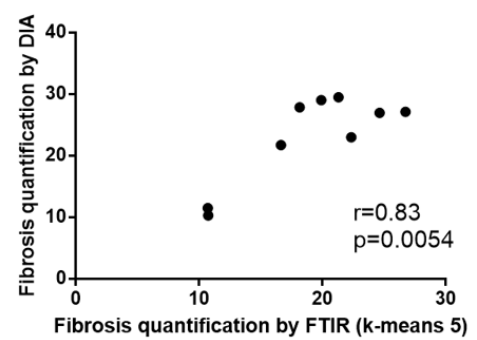

Figure 3. Correlations between fibrosis areas as measured by digital image analysis of histological sections after Masson's trichrome staining and by k-means clustering of FTIR spectral images using 2, 3,4 and 5 classes ((A-D) respectively). An outlier point corresponding to a fibrosis area of $60-70 \%$ was excluded from the analysis (see text and Supplementary Figure S1).

This indicates that FTIR spectral analysis of liver tissue is an effective tool for quantifying the area of fibrosis with the advantages of being label-free and non-destructive. Previous studies have reported interest in FTIR spectral imaging to visualize fibrosis regions in hepatic tissues [21-23] but the correlation with quantification obtained by DIA of the stained sections has not been reported to date.

The mean percentage of fibrosis area in samples from patients with cirrhosis complicated by hepatocellular carcinoma was higher than in samples from patients with uncomplicated cirrhosis. This was observed with both methods of quantification, either DIA of stained histological sections or FTIR spectral imaging analysis based on the k-means method (Figure 4).

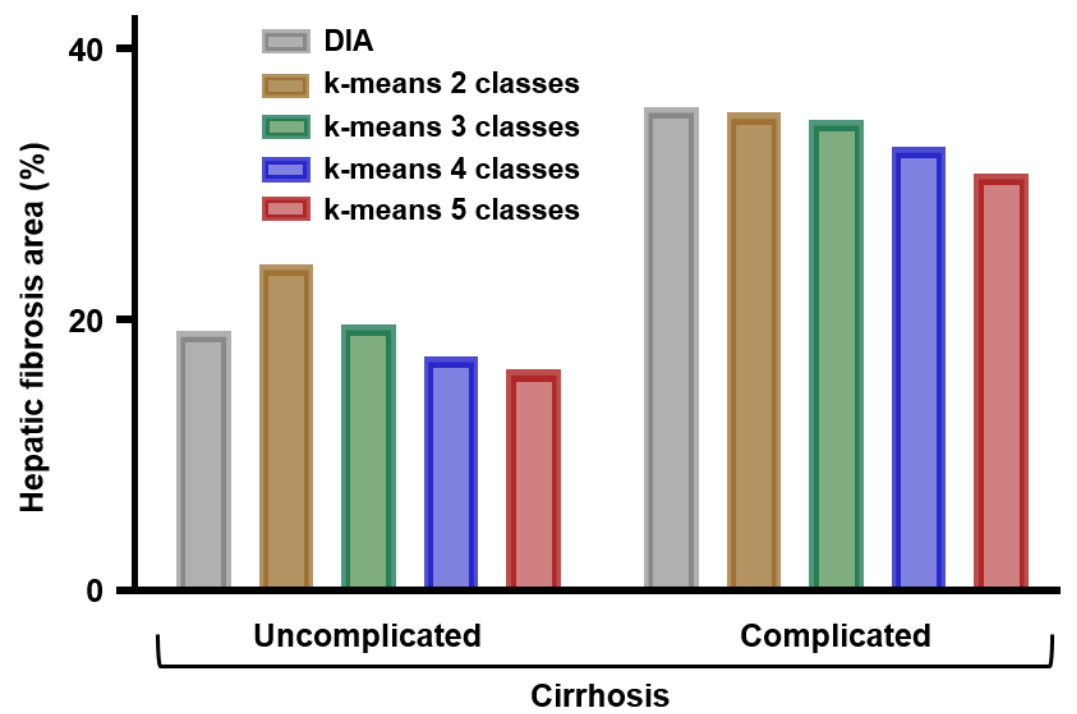

Figure 4. Percentages of fibrosis area in samples from uncomplicated $(n=5)$ and complicated cirrhosis $(n=5)$ as measured by digital image analysis (DIA) of histological sections or by k-means clustering of FTIR spectral images in 2, 3, 4, and 5 classes. 
The small number of cases ( $n=5$ in each group) did not allow a statistical analysis but these data are in accordance with previous studies based on semi-quantitative and quantitative histopathological evaluations of hepatic fibrosis. The hepatic fibrosis area in cirrhotic tissues evaluated by the semi-quantitative histological method has been shown to be correlated with the clinical severity of the disease $[7,8]$ and to be predictive of the risk of liver related complications including hepatocellular carcinoma [9] and of the risk of recurrence after resection of hepatocellular carcinoma [25,26]. In a comparative study, quantitative assessment of hepatic fibrosis by DIA was shown to have a better prognosis than semi-quantitative methods [27]. Thus, FTIR imaging of liver specimens appears as a reliable and easy-to-perform method for automatic quantification of liver fibrosis. Similar observations have been previously reported by our group for quantifying renal interstitial fibrosis in renal allograft biopsy specimens [28].

Analysis of centroids from the k-means clustering with two classes highlights the spectral characteristics of regeneration nodules and fibrosis regions. By visual inspection, marked differences were observed between the two centroid spectra in the $900-1400 \mathrm{~cm}^{-1}$ range as illustrated in Figure $5 \mathrm{~A}$.

A
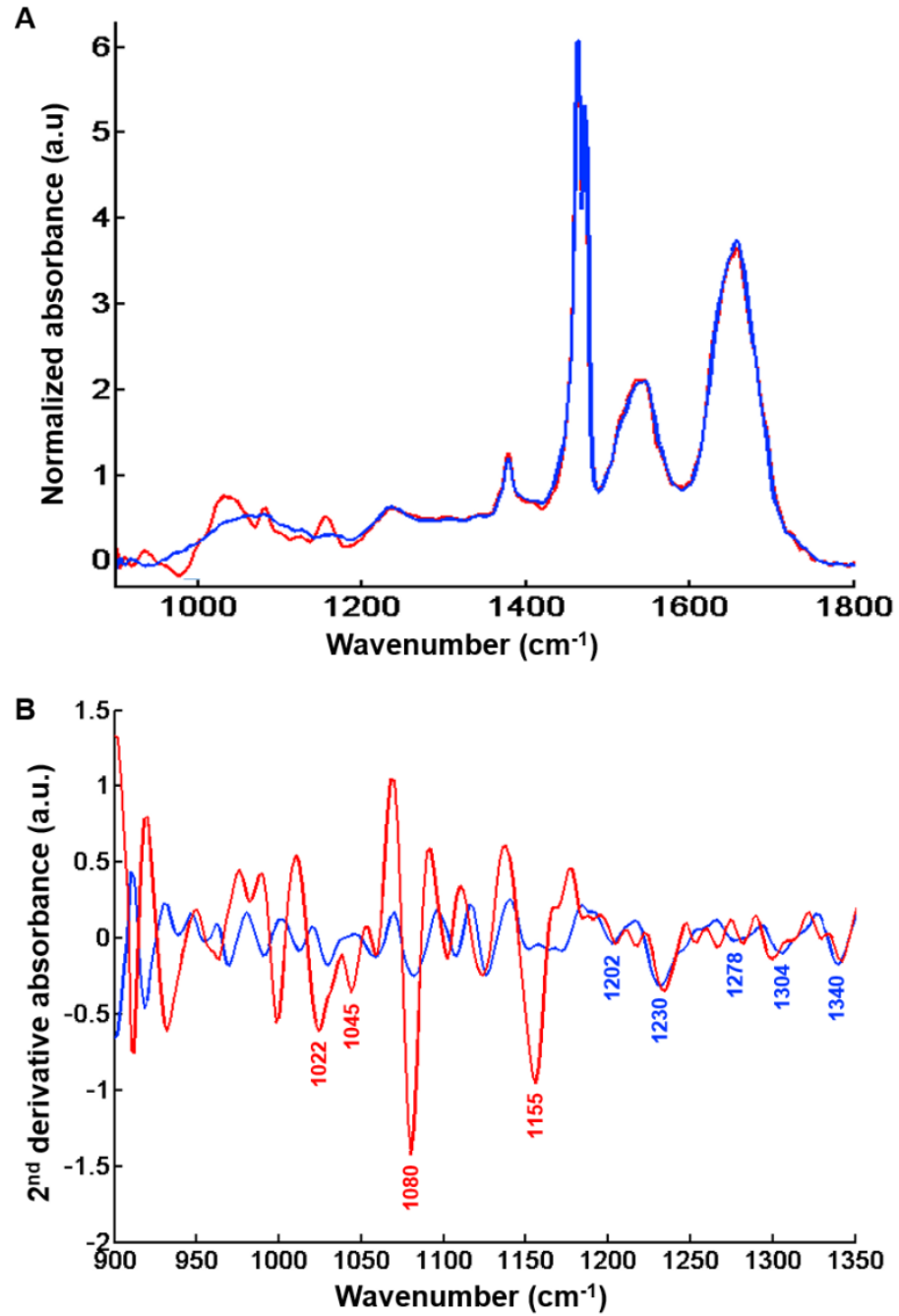

Figure 5. Centroids representative of regeneration nodules and hepatic fibrosis tissue after k-means clustering $(k=2)$ of the spectral image shown in Figure 1C. (A) Centroid spectra representative of fibrosis regions (dark blue line) and regeneration nodules (red line) in the $900-1800 \mathrm{~cm}^{-1}$ spectral range. (B) Second derivative of above centroid spectra and peak assignment with fibrosis (dark blue line) and regeneration nodules (red line) in the $900-1350 \mathrm{~cm}^{-1}$ spectral range. Peaks that can be tentatively assigned to glycogen (regeneration nodules) and collagen (fibrosis tissue) are indicated in red and blue respectively. Results are from FTIR images acquired with a projected pixel size of $25 \mu \mathrm{m}$. 
The centroids corresponding to regeneration nodules exhibit high absorbance levels related to the presence of glycogenated hepatocytes (Figure 5B). Differences in absorption features are mainly linked to glycogen, and observed at $1022 \mathrm{~cm}^{-1}\left(\mathrm{CH}_{2} \mathrm{OH}\right.$ group vibrations), $1045 \mathrm{~cm}^{-1}$ (C-O stretching coupled with C-O bending of the C-OH groups) [21,29-31], $1080 \mathrm{~cm}^{-1}$ (C-C stretch) $[21,32,33]$ and $1155 \mathrm{~cm}^{-1}$ (C-O stretching vibration) [21,31-33].

The centroid spectrum corresponding to fibrosis tissue was characterized by absorbance peaks related to vibrational modes of collagen as evidenced by the peaks at $1174 \mathrm{~cm}^{-1}$ (C-O stretching) [34], $1202 \mathrm{~cm}^{-1}$, $1230 \mathrm{~cm}^{-1}, 1278 \mathrm{~cm}^{-1}, 1304 \mathrm{~cm}^{-1}$ (amide III) [32,35-40] and $1340 \mathrm{~cm}^{-1}\left(\mathrm{CH}_{2}\right.$ of collagen) $[21,36,37,39,41]$.

\subsection{Analysis of Spectral Images of Fibrosis Tissue Areas Acquired with a Projected Pixel Size of $2.7 \mu \mathrm{m}$}

A common K-means clustering with four classes was carried out on 48 spectral images of fibrosis regions from the five samples of uncomplicated cirrhosis ( 25 spectral images) and five samples of cirrhosis with HCC (23 spectral images) on the $1040-1425 \mathrm{~cm}^{-1}$ spectral range. Spectral images are displayed in Figure 6 and cluster centroid spectra in Figure 7A.

A
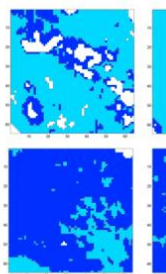

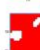
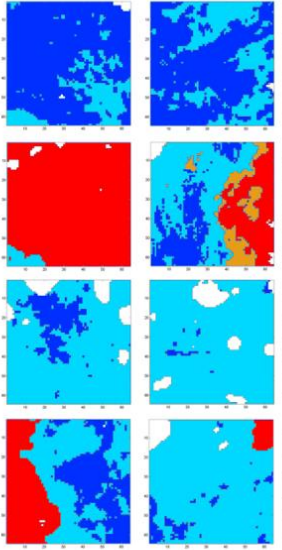

$\mathbf{B}$
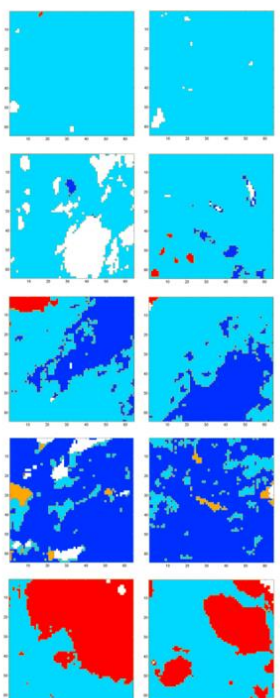
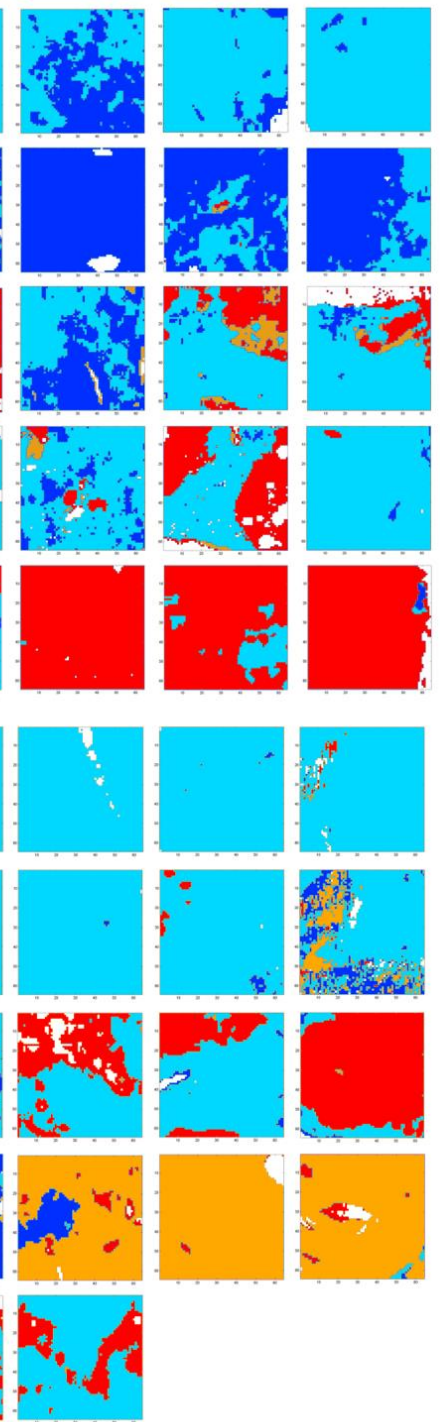

Figure 6. FTIR spectral images of 48 fibrosis areas $\left(172.8 \times 172.8 \mu \mathrm{m}^{2}\right)$ recorded from five samples of uncomplicated cirrhosis (panel (A): 25 images) and five samples of cirrhosis with HCC (panel (B): 23 images) clustered in four classes in the spectral range $1040-1425 \mathrm{~cm}^{-1}$. Results are from FTIR images acquired with a projected pixel size of $2.7 \mu \mathrm{m}$. 

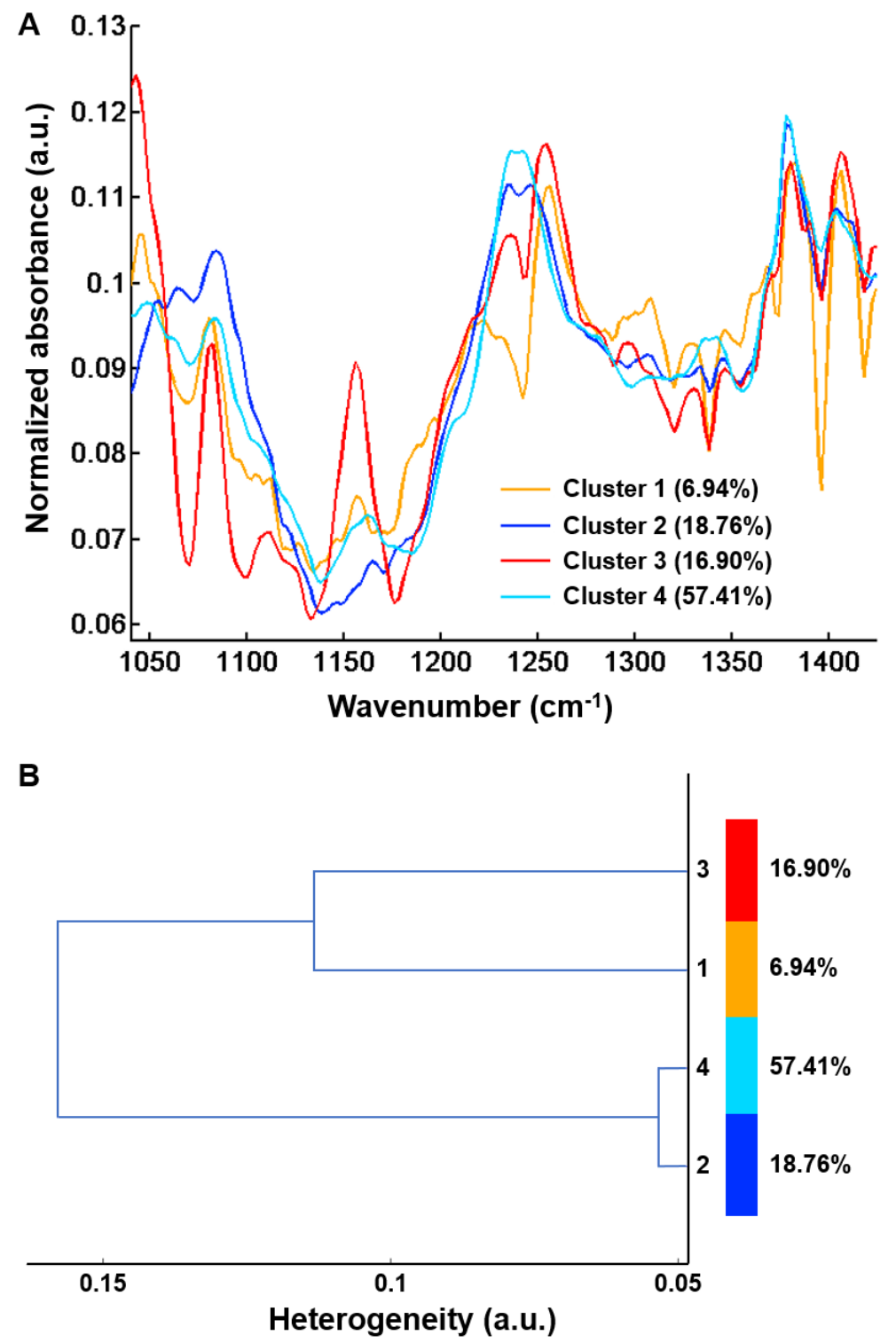

Figure 7. Centroids obtained after common k-means analysis of FTIR spectral images using four classes from 48 hepatic samples without $(n=25)$ and with $(n=23)$ complications in the $1040-1425 \mathrm{~cm}^{-1}$ spectral range. (A) Centroid spectra and (B) Hierarchical cluster analysis of the four centroid spectra. Results are from FTIR images acquired with a projected pixel size of $2.7 \mu \mathrm{m}$.

Although images focused on fibrosis areas, some of them included parts of adjacent regenerative nodules. As shown in Figure 7B, the hierarchical clustering dendrogram clearly shows the formation of two groups. These groups were assigned by second derivative spectral analysis to regeneration nodules (clusters 1 and 3) and fibrosis (clusters 2 and 4) (Figure 8).

Clusters 1 and 3 displayed the characteristic absorption bands of glycogenated hepatocytes as described above at $1044 \mathrm{~cm}^{-1}, 1082 \mathrm{~cm}^{-1}$ and $1156 \mathrm{~cm}^{-1}$ [29-33]. The two other centroid spectra (clusters 2 and 4) were related to the fibrotic tissue. These were characterized by high absorbance levels related to the presence of collagen at $1050 \mathrm{~cm}^{-1}, 1064 \mathrm{~cm}^{-1}, 1084 \mathrm{~cm}^{-1}(\mathrm{C}-\mathrm{O}$ and C-O-C stretching of carbohydrate moieties) [16], $1164 \mathrm{~cm}^{-1}$ (C-O stretching) [42], 1202-1204 cm $\mathrm{cm}^{-1}, 1234 \mathrm{~cm}^{-1}, 1280-1284 \mathrm{~cm}^{-1}$, $1306-1310 \mathrm{~cm}^{-1}$ (amide III) [32,35-40], 1342-1346 cm $\mathrm{cm}^{-1}\left(\mathrm{CH}_{2}\right.$ of collagen) $[21,36,37,39,41]$ and $1404 \mathrm{~cm}^{-1}$ $\left(\mathrm{CH}_{3}\right.$ of collagen $)[16,38]$. 

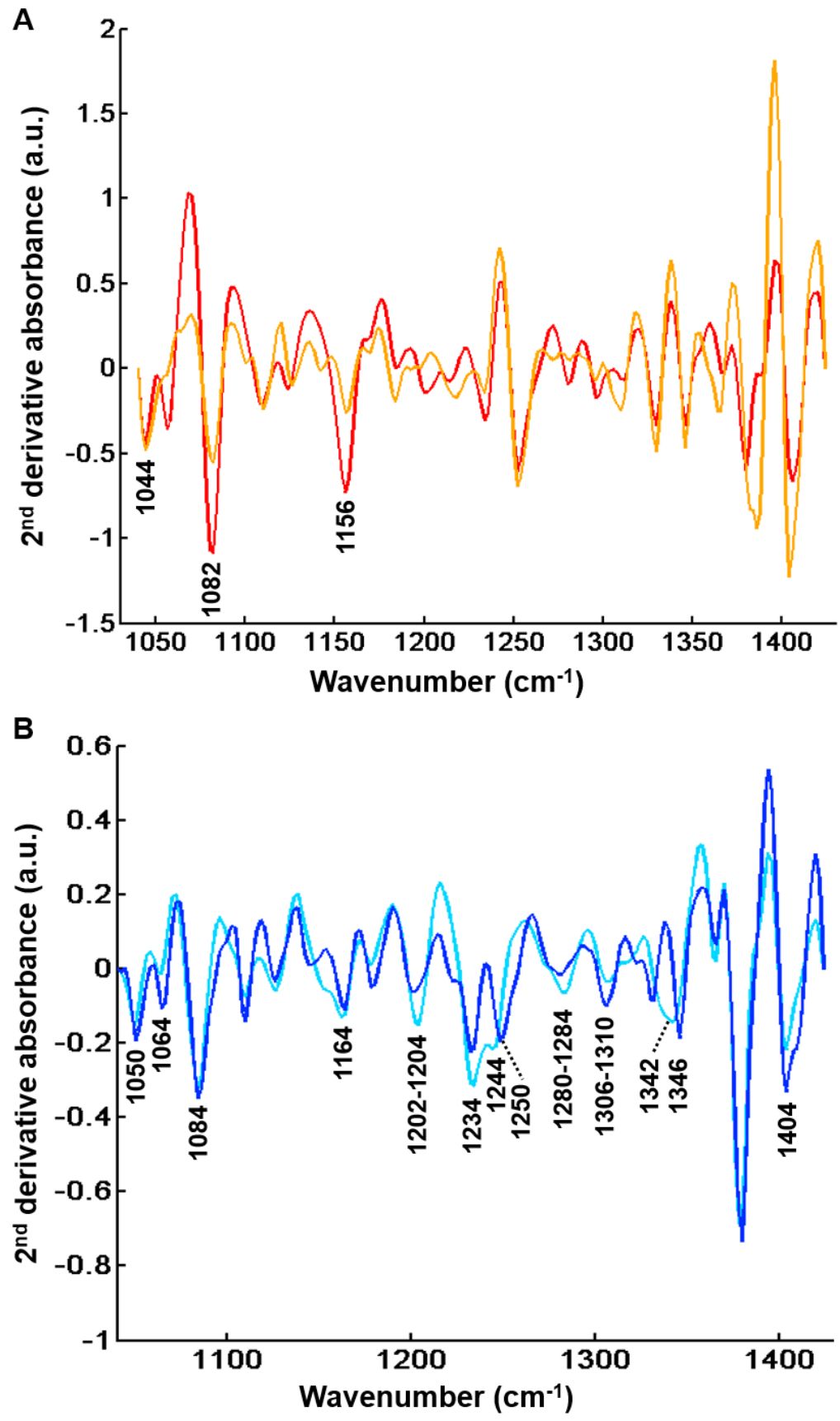

Figure 8. Second derivative centroid spectra after common $k$-means analysis $(k=4)$ of FTIR images from 48 hepatic samples without $(n=25)$ and with $(n=23)$ complications in the spectral range $1040-1425 \mathrm{~cm}^{-1}$. (A) Centroids corresponding to regeneration nodules. Peaks that can be tentatively assigned to glycogen are indicated (B) Centroids corresponding to fibrosis regions. Peaks that can be tentatively associated to collagen are indicated. Results are from FTIR images acquired with a projected pixel size of $2.7 \mu \mathrm{m}$.

Of interest, the distribution of the two clusters within the fibrosis tissue appeared different between the two groups of liver specimens corresponding to early uncomplicated and late complicated stages of cirrhosis (Table 1). Although this is an interesting finding, the statistical analysis was considered inappropriate as the number of fibrosis areas was small and not equal for each patient, and it is therefore not presented. 
Table 1. Comparison of the distribution of the two spectral classes corresponding to fibrosis areas from uncomplicated cirrhosis and cirrhosis with hepatocellular carcinoma (HCC).

\begin{tabular}{ccc}
\hline & \multicolumn{2}{c}{ Fibrosis Areas (\% of Total Tissue) } \\
\hline Spectral Classes of Fibrosis & Uncomplicated Cirrhosis & Cirrhosis with HCC \\
\hline Cluster 4 & $69.4 \%$ & $83.9 \%$ \\
\hline Cluster 2 & $30.6 \%$ & $16.1 \%$ \\
\hline
\end{tabular}

Nevertheless, as shown in Table 1, the proportion of fibrosis tissue in cluster 4 was higher in complicated cirrhosis than in uncomplicated cirrhosis. Although definitive and statistically-based conclusions cannot be drawn, our results tend to support the hypothesis that fibrosis tissue in advanced complicated cirrhosis exhibits specific biomolecular and structural characteristics detectable by FTIR imaging that may be used to identify different stages of cirrhosis with increasing risk of clinical complications. These preliminary results are worth exploring in a larger data set. Compared with cluster 2, cluster 4 is characterized either by higher absorbance peaks or by a modification in spectral profile $\left(1204,1234,1244,1284\right.$ and $\left.1342 \mathrm{~cm}^{-1}\right)$. The higher absorption in amide III peaks $(1204,1234$ and $1284 \mathrm{~cm}^{-1}$ ) may be explained by the remodeling of extracellular matrix with modifications of secondary structure of the collagen fibers. It is noteworthy that spectral modifications have been reported in an animal model of collagen aging using FTIR spectroscopy. It was shown that the spectral profile of collagen I from old rat tail tendons was characterized by an increase in absorption in the $1200-1300 \mathrm{~cm}^{-1}$ amide III region compared with newborns and young adults [19]. This is concordant with the hypothesis that changes in amide III peak absorption observed in cluster 4 may be related to a more advanced stage of fibrosis where crosslinking could modify the organization of collagen in extracellular matrix. In addition, spectral downshifts were observed at 1244 and $1342 \mathrm{~cm}^{-1}$ in cluster 4 compared with 1250 and $1346 \mathrm{~cm}^{-1}$ in cluster 2, respectively, which could also be related to structural and molecular constraints as a consequence of collagen crosslinks. Altogether, these results indicate that qualitative spectral changes reflecting the composition and structure of hepatic fibrosis could be useful markers of the severity of cirrhosis. With the advent of quantum cascade lasers for IR imaging and with an a priori knowledge of the specific spectral markers bands, these can be tuned to perform fast high-throughput screening of biopsies, thus overcoming the slow acquisition speed of FTIR-based infrared microscopes, which is a drawback for routine applications in clinics [21,43].

\section{Materials and Methods}

\subsection{Selection and Preparation of Samples}

The study was conducted on percutaneous liver biopsies and surgical liver samples from cirrhotic patients, stored in the tissue bank of the Laboratory of Biopathology of the Reims University Hospital. These samples were initially formalin fixed and paraffin embedded (FFPE) for routine histopathological analysis. Two groups of samples were selected: five from cirrhotic patients without complication with a follow-up of 10 years after the specimen collection and five from patients with cirrhosis complicated by an HCC. In patients with HCC, the study was performed on cirrhotic tissue located at a distance from the tumor.

Two adjacent $5 \mu \mathrm{m}$ thick sections from selected FFPE blocks were obtained using a microtome (Leica Biosystem, Newcastle, UK). The first section was deposited on a glass slide for histology and the second on a calcium fluoride $\left(\mathrm{CaF}_{2}\right)$ window for FTIR imaging. Patient records and tissue specimens were anonymized and de-identified prior to analysis. This procedure was conducted in accordance with the reference methodology MR004 of the "Commission Nationale de l'Informatique et des Libertés" (n²206749, 13/09/2018). 


\subsection{Histological and Digital Analysis}

For histology, Masson's trichrome was used. It stains the cytoplasm in pink, the nuclei in black and highlights the collagen fibers in green (Figures 1A and 2A). It was used to locate and quantify the area of fibrosis after digital scanning of these slides (iScan Coreo, Roche Ventana, Meillan, France) (Figures 1B and 2B). The quantification was based on DIA using the public domain National Institutes of Health (NIH) Image program ImageJ (available at http://rsb.info.nih.gov/nih-image/). In this process, the histological image was binarized to eliminate the background of pure paraffin. Then, a color deconvolution in three channels was performed allowing the identification of collagen. The ratio of the fibrosis area to the total area gave the percentage of fibrosis in the sample.

\subsection{FTIR Data Collection}

\subsubsection{Infrared Imaging of Whole Hepatic Samples}

FTIR imaging acquisitions of whole hepatic samples were obtained using the Spectrum Spotlight ${ }^{\mathrm{TM}}$ 400 microscope coupled to a Spectrum One FTIR spectrometer (Perkin Elmer, Villebon sur Yvette, France). This device was equipped with a liquid nitrogen-cooled, 16-pixel line mercury cadmium telluride (MCT) detector for imaging. The pixel size used was $25 \times 25 \mu \mathrm{m}^{2}$. Spectra were recorded in the $4000-650 \mathrm{~cm}^{-1}$ range with 8 accumulations per pixel at a resolution of $4 \mathrm{~cm}^{-1}$. Prior to image acquisition, a background spectrum was recorded on a clean surface of the $\mathrm{CaF}_{2}$ window with 90 accumulations at $4 \mathrm{~cm}^{-1}$ spectral resolution and automatically removed from each pixel spectrum using the Spectrum Image software (Perkin Elmer, Waltham, MA, USA). In addition, an image of pure paraffin was collected at the periphery of the tissue with the same parameters and used for digital deparaffinisation in the extended multiplicative signal correction (EMSC) model.

\subsubsection{FTIR Imaging Focused on Hepatic Fibrosis Tissue}

In order to analyze more precisely the spectral characteristics of hepatic fibrosis, FTIR imaging was acquired with a projected pixel size of $2.7 \mu \mathrm{m}$ in fibrosis tissue of each of the 10 samples. For this purpose, a Vertex 70 FTIR spectrometer coupled to a Hyperion 3000 microscope (Bruker Optics, Etlingen, Germany) was employed. It was equipped with a liquid nitrogen-cooled $64 \times 64$ pixels focal plane array MCT detector. Using a Cassegrain $\times 15$ objective, a pixel resolution of $2.7 \times 2.7 \mu \mathrm{m}^{2}$ was obtained. Spectral images were acquired on the 3900-900 $\mathrm{cm}^{-1}$ range with 512 accumulations per spectrum at a resolution of $4 \mathrm{~cm}^{-1}$. As above, prior to image acquisition, a background spectrum was recorded on a clean surface of the $\mathrm{CaF}_{2}$ window with 512 accumulations at $4 \mathrm{~cm}^{-1}$ resolution and automatically removed from each pixel spectrum using the OPUS software (Version 7.2, Bruker Optics, Etlingen, Germany). For digital dewaxing, a pure paraffin image was collected at the periphery of the tissue using the same measurement conditions.

\subsection{Spectral Data Preprocessing}

Image preprocessing was performed using in-house algorithms written in Matlab (The Mathworks, Natick, MA, USA). Digital dewaxing was done by the EMSC method. This correction was applied to all spectral images in order to neutralize the paraffin spectrum in the IR spectra as previously reported by our group [44,45]. This EMSC model also eliminates outlier spectra, corrects scattering effects in FTIR spectra and normalizes them.

\subsection{Analysis of Spectral Images of Whole Hepatic Samples}

After the EMSC preprocessing, k-means clustering [46] was applied on FTIR images for regrouping the spectra according to their spectral similarity in $\mathrm{k}$ classes. In this procedure, each class is represented by a centroid spectrum that is the class mean spectrum. The k-means procedure is unsupervised and starts by selecting random $\mathrm{k}$ spectra as centroids and then allocating every spectrum of the spectral 
image to the nearest centroid using Euclidian distance as a metric. Each centroid is then readjusted and this operation is repeated until a stable partition is reached. To visualize the k-means clustering results, a different color is randomly assigned to each class and a reconstruction of the spectral image is performed. In our study, k-means clustering was used to partition each FTIR spectral image in 2,3, 4 and 5 spectral classes using the $900-1800 \mathrm{~cm}^{-1}$ fingerprint region. All these spectral images were compared to their adjacent tissue section stained with Masson's trichrome in order to annotate the spectral classes to their corresponding histological structures, particularly the fibrotic tissue. K-means clustering gave the percentage of the class(es) corresponding to fibrosis. Fibrosis area in histological sections was quantified by DIA as previously described.

In addition, the centroid spectra were analyzed to identify the discriminant IR absorption peaks corresponding to histological structures. They were derived to the second order to better visualize and assign overlapping peaks.

\subsection{Analysis of Spectral Images Focused on Fibrosis Areas}

Comparison of spectral features of fibrosis between samples from uncomplicated and complicated cirrhosis was performed using spectral images with a higher spatial resolution. FTIR imaging with a pixel resolution of $2.7 \times 2.7 \mu \mathrm{m}^{2}$ was performed on fibrosis tissue of $172.8 \times 172.8 \mu \mathrm{m}^{2}$. Five fibrosis regions were selected for each sample except one where only three zones were available for analysis. Finally, 48 regions ( 25 from uncomplicated cirrhosis and 23 from complicated cirrhosis) were analyzed using a common k-means classification in four spectral classes on the $1040-1425 \mathrm{~cm}^{-1}$ spectral window, chosen as it includes most FTIR absorption peaks of collagen [20] and excludes paraffin peaks. In this procedure, spectral classes are common to all analyzed images, making it possible to compare the distribution of spectral characteristics between different samples. In common k-means image clustering, the same spectral class is represented by the same color coding.

\subsection{Statistical Analysis}

Pearson's correlation coefficient was used to evaluate the correlation between the percentages of fibrosis areas in the whole tissue as measured on spectral images and on histological sections.

\section{Conclusions}

Our study shows that FTIR imaging of cirrhotic tissues allows the differentiation of regenerative nodules and fibrosis tissue and is effective for quantifying fibrosis area with the advantage of being label free and non-destructive. In addition, spectral image analysis with a higher spatial resolution highlights the qualitative changes of fibrosis tissue during the course of cirrhosis, differentiating liver fibrosis from complicated and uncomplicated cirrhosis. Future development would be to build a predictive model based on fibrosis tissue FTIR spectral characteristics allowing the subclassification of cirrhosis in different stages of severity corresponding to an increasing risk of complications such as HCC.

Supplementary Materials: The following are available online. Figure S1: Correlations between fibrosis areas as measured by digital image analysis of histological sections after Masson's trichrome staining and by k-means clustering of FTIR spectral images using 2, 3, 4 and 5 classes (A, B, C and D respectively). The scatter plots include the outlier point corresponding to a fibrosis area of $60-70 \%$.

Author Contributions: Conceptualization, G.T. and G.D.S.; Methodology, J.M., P.B., V.U., R.G., G.D.S. and G.T.; Software, C.G. and C.T.; Validation, V.U., R.G., G.D.S. and G.T.; Formal Analysis, J.M., P.B., J.G, V.U., C.G., C.T., R.G., A.M., G.D.S. and G.T.; Investigation, J.M., P.B., J.G., V.U.; Resources, A.M.; Writing-Original Draft Preparation, J.M., P.B. and G.T.; Writing-Review \& Editing: all authors; Supervision, G.D.S. and G.T. All authors have read and agreed to the published version of the manuscript.

Funding: This research received no external funding.

Acknowledgments: The authors thank the PICT-IBiSA Platform for vibrational spectroscopy instrument facilities and Nicole Bouland for technical assistance in histological preparations. 
Conflicts of Interest: The authors declare no conflict of interest.

\section{References}

1. Anthony, P.P.; Ishak, K.G.; Nayak, N.C.; Poulsen, H.E.; Scheuer, P.J.; Sobin, L.H. The morphology of cirrhosis: Definition, nomenclature, and classification. Bull. World Health Organ. 1977, 55, 521-540.

2. The French, M.C.S.G. Intraobserver and interobserver variations in liver biopsy interpretation in patients with chronic hepatitis C. Hepatology 1994, 20, 15-20.

3. Ishak, K.; Baptista, A.; Bianchi, L.; Callea, F.; De Groote, J.; Gudat, F.; Denk, H.; Desmet, V.; Korb, G.; MacSween, R.N.M. Histological grading and staging of chronic hepatitis. J. Hepatol. 1995, 22, 696-699. [CrossRef]

4. Pellicoro, A.; Ramachandran, P.; Iredale, J.P. Reversibility of liver fibrosis. Fibrogenesis Tissue Repair 2012, 5 (Suppl. 1), S26. [CrossRef]

5. Kutami, R.; Girgrah, N.; Wanless, I.R.; Sniderman, K.; Wong, F.S.; Sherman, M.; Heathcote, J.; Lilly, L. The Laennec grading system for assessment of hepatic fibrosis: Validation by correlation with wedged hepatic vein pressure and clinical features. Hepatology 2000, 32, 407A.

6. Wanless, I.R.; Sweeney, G.; Dhillon, A.P.; Guido, M.; Piga, A.; Galanello, R.; Gamberini, M.R.; Schwartz, E.; Cohen, A.R. Lack of progressive hepatic fibrosis during long-term therapy with deferiprone in subjects with transfusion-dependent beta-thalassemia. Blood 2002, 100, 1566-1569. [CrossRef]

7. Kim, M.Y.; Cho, M.Y.; Baik, S.K.; Park, H.J.; Jeon, H.K.; Im, C.K.; Won, C.S.; Kim, J.W.; Kim, H.S.; Kwon, S.O. Histological subclassification of cirrhosis using the Laennec fibrosis scoring system correlates with clinical stage and grade of portal hypertension. J. Hepatol. 2011, 55, 1004-1009. [CrossRef]

8. Kim, G.; Lee, S.S.; Baik, S.K.; Cho, Y.Z.; Kim, M.Y.; Kwon, S.O.; Cha, S.H.; Cho, M.Y. The need for histological subclassification of cirrhosis: A systematic review and meta-analysis. Liver Int. 2016, 36, 847-855. [CrossRef]

9. Kim, S.U.; Oh, H.J.; Wanless, I.R.; Lee, S.; Han, K.-H.; Park, Y.N. The Laennec staging system for histological sub-classification of cirrhosis is useful for stratification of prognosis in patients with liver cirrhosis. J. Hepatol. 2012, 57, 556-563. [CrossRef]

10. Gressner, A.M.; Weiskirchen, R. Modern pathogenetic concepts of liver fibrosis suggest stellate cells and TGF-beta as major players and therapeutic targets. J. Cell Mol. Med. 2006, 10, 76-99. [CrossRef] [PubMed]

11. Karsdal, M.A.; Nielsen, S.H.; Leeming, D.J.; Langholm, L.L.; Nielsen, M.J.; Manon-Jensen, T.; Siebuhr, A.; Gudmann, N.S.; Ronnow, S.; Sand, J.M.; et al. The good and the bad collagens of fibrosis-their role in signaling and organ function. Adv. Drug. Deliv. Rev. 2017, 121, 43-56. [CrossRef] [PubMed]

12. Friedman, S.L. Mechanisms of hepatic fibrogenesis. Gastroenterology 2008, 134, 1655-1669. [CrossRef] [PubMed]

13. Grenard, P.; Bresson-Hadni, S.; El Alaoui, S.; Chevallier, M.; Vuitton, D.A.; Ricard-Blum, S. Transglutaminase-mediated cross-linking is involved in the stabilization of extracellular matrix in human liver fibrosis. J. Hepatol. 2001, 35, 367-375. [CrossRef]

14. Ricard-Blum, S.; Baffet, G.; Théret, N. Molecular and tissue alterations of collagens in fibrosis. Matrix Biol. 2018, 68-69, 122-149. [CrossRef]

15. Baker, M.J.; Trevisan, J.; Bassan, P.; Bhargava, R.; Butler, H.J.; Dorling, K.M.; Fielden, P.R.; Fogarty, S.W.; Fullwood, N.J.; Heys, K.A. Using Fourier transform IR spectroscopy to analyze biological materials. Nat. Protoc. 2014, 9, 1771-1791. [CrossRef]

16. Belbachir, K.; Noreen, R.; Gouspillou, G.; Petibois, C. Collagen types analysis and differentiation by FTIR spectroscopy. Anal. Bioanal. Chem. 2009, 395, 829-837. [CrossRef]

17. Petibois, C.; Gouspillou, G.; Wehbe, K.; Delage, J.-P.; Déléris, G. Analysis of type I and IV collagens by FT-IR spectroscopy and imaging for a molecular investigation of skeletal muscle connective tissue. Anal. Bioanal. Chem. 2006, 386, 1961-1966. [CrossRef]

18. Riaz, T.; Zeeshan, R.; Zarif, F.; Ilyas, K.; Muhammad, N.; Safi, S.Z.; Rahim, A.; Rizvi, S.A.A.; Rehman, I.U. FTIR analysis of natural and synthetic collagen. Appl. Spectrosc. Rev. 2018, 53, 703-746. [CrossRef]

19. Guilbert, M.; Eklouh-Molinier, C.; Wehbe, K.; Sulé-Suso, J.; Yang, Y.; Cinque, G.; Jeannesson, P.; Sockalingum, G.D. Probing single-tumor cell interactions with different-age type I collagen networks by synchrotron-based Fourier transform infrared microspectroscopy. J. Biomed. Opt. 2014, 19, 111612. [CrossRef] 
20. Guilbert, M.; Said, G.; Happillon, T.; Untereiner, V.; Garnotel, R.; Jeannesson, P.; Sockalingum, G.D. Probing non-enzymatic glycation of type I collagen: A novel approach using Raman and infrared biophotonic methods. Biochim. Biophys. Acta 2013, 1830, 3525-3531. [CrossRef]

21. Bird, B.; Rowlette, J. A protocol for rapid, label-free histochemical imaging of fibrotic liver. Analyst 2017, 142, 1179-1184. [CrossRef]

22. Crombie, D.E.; Mackay, I.R.; Wood, B.R.; McNaughton, D.; Rowley, M.J. Hepatobiliary and pancreatic: Liver histopathology: Fourier transform infrared microspectroscopic imaging for objective and quantifiable assessment of liver biopsies. J. Gastroenterol. Hepatol. 2005, 20, 485. [CrossRef]

23. Le Naour, F.; Sandt, C.; Peng, C.; Trcera, N.; Chiappini, F.; Flank, A.-M.; Guettier, C.; Dumas, P. In situ chemical composition analysis of cirrhosis by combining synchrotron fourier transform infrared and synchrotron $\mathrm{X}$-ray fluorescence microspectroscopies on the same tissue section. Anal. Chem. 2012, 84, 10260-10266. [CrossRef]

24. Sreedhar, H.; Varma, V.K.; Gambacorta, F.V.; Guzman, G.; Walsh, M.J. Infrared spectroscopic imaging detects chemical modifications in liver fibrosis due to diabetes and disease. Biomed. Opt. Express 2016, 7, 2419-2424. [CrossRef]

25. Huang, Z.; Liang, B.; Xiong, M.; Dong, K.; Zhang, Z.; Zhang, E.; Li, C.; Chen, X. Severity of cirrhosis should determine the operative modality for patients with early hepatocellular carcinoma and compensated liver function. Surgery 2016, 159, 621-631. [CrossRef]

26. Kim, S.U.; Jung, K.S.; Lee, S.; Park, J.Y.; Kim, D.Y.; Ahn, S.H.; Choi, G.H.; Kim, K.S.; Choi, J.S.; Han, K. Histological subclassification of cirrhosis can predict recurrence after curative resection of hepatocellular carcinoma. Liver Int. 2014, 34, 1008-1017. [CrossRef]

27. Tsochatzis, E.; Bruno, S.; Isgro, G.; Hall, A.; Theocharidou, E.; Manousou, P.; Dhillon, A.P.; Burroughs, A.K.; Luong, T.V. Collagen proportionate area is superior to other histological methods for sub-classifying cirrhosis and determining prognosis. J. Hepatol. 2014, 60, 948-954. [CrossRef]

28. Vuiblet, V.; Fere, M.; Gobinet, C.; Birembaut, P.; Piot, O.; Rieu, P. Renal graft fibrosis and inflammation quantification by an automated Fourier-transform infrared imaging technique. J. Am. Soc. Nephrol. 2016, 27, 2382-2391. [CrossRef]

29. Huleihel, M.; Salman, A.; Erukhimovitch, V.; Ramesh, J.; Hammody, Z.; Mordechai, S. Novel spectral method for the study of viral carcinogenesis in vitro. J. Biochem. Biophys. Methods 2002, 50, 111-121. [CrossRef]

30. Mordechai, S.; Mordehai, J.; Ramesh, J.; Levi, C.; Huleihal, M.; Erukhimovitch, V.; Moser, A.; Kapelushnik, J. Application of FTIR microspectroscopy for the follow-up of childhood leukemia chemotherapy. In Proceedings of the Subsurface and Surface Sensing Technologies and Applications III, San Diego, CA, USA, 27 November 2001. [CrossRef]

31. Wood, B.R.; Quinn, M.A.; Tait, B.; Ashdown, M.; Hislop, T.; Romeo, M.; McNaughton, D. FTIR microspectroscopic study of cell types and potential confounding variables in screening for cervical malignancies. Biospectroscopy 1998, 4, 75-91. [CrossRef]

32. Chiriboga, L.; Xie, P.; Yee, H.; Vigorita, V.; Zarou, D.; Zakim, D.; Diem, M. Infrared spectroscopy of human tissue. I. Differentiation and maturation of epithelial cells in the human cervix. Biospectroscopy 1998, 4, 47-53. [CrossRef]

33. Wong, P.T.; Wong, R.K.; Caputo, T.A.; Godwin, T.A.; Rigas, B. Infrared spectroscopy of exfoliated human cervical cells: Evidence of extensive structural changes during carcinogenesis. Proc. Natl. Acad Sci. USA 1991, 88, 10988-10992. [CrossRef] [PubMed]

34. Fukuyama, Y.; Yoshida, S.; Yanagisawa, S.; Shimizu, M. A study on the differences between oral squamous cell carcinomas and normal oral mucosas measured by Fourier transform infrared spectroscopy. Biospectroscopy 1999, 5, 117-126. [CrossRef]

35. Eckel, R.; Huo, H.; Guan, H.-W.; Hu, X.; Che, X.; Huang, W.-D. Characteristic infrared spectroscopic patterns in the protein bands of human breast cancer tissue. Vib. Spectrosc. 2001, 27, 165-173. [CrossRef]

36. Fabian, H.; Wessel, R.; Jackson, M.; Schwartz, A.; Lasch, P.; Fichtner, I.; Mantsch, H.H.; Naumann, D. IR spectroscopy and IR microscopy of human breast tumors, xenografted breast tumors, and breast tumor cell lines. In Proceedings of the Infrared Spectroscopy: New Tool in Medicine, San Jose, CA, USA, 24 April 1998. [CrossRef]

37. Fujioka, N.; Morimoto, Y.; Arai, T.; Kikuchi, M. Discrimination between normal and malignant human gastric tissues by Fourier transform infrared spectroscopy. Cancer Detect. Prev. 2004, 28, 32-36. [CrossRef] 
38. Fung, M.F.K.; Senterman, M.K.; Mikhael, N.Z.; Lacelle, S.; Wong, P.T.T. Pressure-tuning Fourier transform infrared spectroscopic study of carcinogenesis in human endometrium. Biospectroscopy 1996, 2, 155-165. [CrossRef]

39. McIntosh, L.M.; Jackson, M.; Mantsch, H.H.; Stranc, M.F.; Pilavdzic, D.; Crowson, A.N. Infrared spectra of basal cell carcinomas are distinct from non-tumor-bearing skin components. J. Invest. Derm. 1999, 112, 951-956. [CrossRef]

40. Richter, T.; Steiner, G.; Abu-Id, M.H.; Salzer, R.; Bergmann, R.; Rodig, H.; Johannsen, B. Identification of tumor tissue by FTIR spectroscopy in combination with positron emission tomography. Vib. Spectrosc. 2002, 28, 103-110. [CrossRef]

41. Andrus, P.G.L.; Strickland, R.D. Cancer grading by Fourier transform infrared spectroscopy. Biospectroscopy 1998, 4, 37-46. [CrossRef]

42. Rigas, B.; Morgello, S.; Goldman, I.S.; Wong, P.T. Human colorectal cancers display abnormal Fourier-transform infrared spectra. Proc. Natl. Acad. Sci. USA 1990, 87, 8140-8144. [CrossRef]

43. Mittal, S.; Yeh, K.; Leslie, L.S.; Kenkel, S.; Kajdacsy-Balla, A.; Bhargava, R. Simultaneous cancer and tumor microenvironment subtyping using confocal infrared microscopy for all-digital molecular histopathology. Proc. Natl. Acad. Sci. USA 2018, 115, E5651-E5660. [CrossRef] [PubMed]

44. Ly, E.; Piot, O.; Wolthuis, R.; Durlach, A.; Bernard, P.; Manfait, M. Combination of FTIR spectral imaging and chemometrics for tumour detection from paraffin-embedded biopsies. Analyst 2008, 133, 197-205. [CrossRef] [PubMed]

45. Wolthuis, R.; Travo, A.; Nicolet, C.; Neuville, A.; Gaub, M.-P.; Guenot, D.; Ly, E.; Manfait, M.; Jeannesson, P.; Piot, O. IR spectral imaging for histopathological characterization of xenografted human colon carcinomas. Anal. Chem. 2008, 80, 8461-8469. [CrossRef] [PubMed]

46. Jain, A.K.; Dubes, R.C. Algorithms for Clustering Data; Prentice-Hall, Inc.: Upper Saddle River, NJ, USA, 1988.

Sample Availability: Samples of the compounds are not available from the authors.

(C) 2020 by the authors. Licensee MDPI, Basel, Switzerland. This article is an open access article distributed under the terms and conditions of the Creative Commons Attribution (CC BY) license (http://creativecommons.org/licenses/by/4.0/). 